\title{
Efficiency and stability of probabilistic assignments in marriage problems
}

\author{
Battal Doğan ${ }^{\mathrm{a}}$, Kemal Yıldız ${ }^{\mathrm{b}, *}$ \\ a Faculty of Business and Economics, University of Lausanne, Switzerland \\ ${ }^{\mathrm{b}}$ Department of Economics, Bilkent University, Turkey
}

\section{A R T I C L E I N F O}

\section{Article history:}

Received 20 July 2014

Available online 17 December 2015

\section{JEL classification:}

C60

C71

C78

D61

\section{Keywords:}

Marriage problems

Probabilistic assignment

Efficiency

Stability

\begin{abstract}
A B S T R A C T
We study marriage problems where two groups of agents, men and women, match each other and probabilistic assignments are possible. When only ordinal preferences are observable, stochastic dominance efficiency (sd-efficiency) is commonly used. First, we provide a characterization of sd-efficient allocations in terms of a property of an order relation defined on the set of man-woman pairs. Then, using this characterization, we constructively prove that for each probabilistic assignment that is sd-efficient for some ordinal preferences, there is a von Neumann-Morgenstern utility profile consistent with the ordinal preferences for which the assignment is Pareto efficient. Second, we show that when the preferences are strict, for each ordinal preference profile and each expost stable probabilistic assignment, there is a von Neumann-Morgenstern utility profile, consistent with the ordinal preferences, for which the assignment belongs to the core of the associated transferable utility game.
\end{abstract}

(c) 2015 Elsevier Inc. All rights reserved.

\section{Introduction}

The theory of two-sided matching problems has been useful in providing solutions to many real-life economic problems (see Roth and Sotomayor, 1990). A marriage problem, which constitutes a basis for two-sided matching problems, consists of two equal-sized groups of agents: men and women. Each man has ordinal preferences over women and vice versa. A majority of the literature is focused on deterministic assignments, where men and women are matched one-to-one. One can also think of probabilistic assignments, which are lotteries over deterministic assignments, and these are interesting for at least two reasons: (1) agents may match in fractions; for example, a consultant may allocate his time among several firms, ${ }^{1}$ (2) probabilistic assignments may help us achieve fairness when it is not possible with deterministic assignments (e.g. Bogomolnaia and Moulin, 2001). Here, we consider probabilistic assignments, and in particular their "stability" and "efficiency".

We consider a model where only ordinal preference information is available. That is, for each man, all we know is his preference ordering over women, and vice versa, which is in line with the applications and the theoretical literature. Most allocation rules that have been discussed in this literature elicit only ordinal preferences, as opposed to utility information

\footnotetext{
We are grateful to Paulo Barelli, Srihari Govindan, Bettina Klaus, Efe Ok, Ariel Rubinstein, William Thomson, Rakesh Vohra, two anonymous referees, the associate editor, seminar participants at Bilkent University and University of Rochester, and participants at the Bosphorous Workshop on Economic Design and Workshop on Social Choice and Mechanism Design at the University of Manchester for their helpful comments.

* Corresponding author.

E-mail addresses: battaldogan@gmail.com (B. Doğan), kemal.yildiz@bilkent.edu.tr (K. Yıldız).

1 See Manjunath (2014) and Bogomolnaia and Moulin (2004) for more examples of fractional matching markets.
} 
over possible mates. ${ }^{2}$ When only ordinal preference information is available, extensively studied efficiency and stability notions are stochastic dominance efficiency (sd-efficiency) and ex-post stability. We ask whether probabilistic assignments that are sd-efficient or ex-post stable for ordinal preferences are possibly efficient or stable for cardinal preferences.

The main contribution of this paper pertains to stability, which is a central robustness condition for two-sided matchings. A deterministic assignment is stable if no pair of a man and a woman prefers each other to their assigned mates. A probabilistic assignment is ex-post stable if it can be expressed as a lottery over stable deterministic assignments. In the deterministic case, if an assignment is stable, then it is also in the core of an associated non-transferable utility (NTU) game (Roth and Sotomayor, 1990). It follows that in an ordinal environment where monetary transfers are not allowed, if an assignment is ex-post stable, then there is no incentive for ex-post ${ }^{3}$ group deviation. However, ex-ante, ${ }^{4}$ a group of men and women may break away from the rest of society and may form matches among themselves that make each of them better off in terms of expected utility. This possibility motivates us to ask whether ex-post stability implies welfare properties deducible from the ordinal preferences that would prevent such break aways.

We show that when preferences are strict (no agent is indifferent between two different agents), for each ex-post stable probabilistic assignment, there is a utility profile consistent with the ordinal preferences such that no group of agents consisting of equal numbers of men and women can deviate to a probabilistic assignment among themselves and make each member better off. In fact, we prove an even stronger result (Theorem 2): for each ex-post stable probabilistic assignment, there is a utility profile consistent with the ordinal preferences such that no group of agents consisting of equal numbers of men and women can deviate to a probabilistic assignment among themselves in which the sum of their expected utilities is greater. Put differently, we associate with each utility profile a transferable utility (TU) game, as in Shapley and Shubik (1971), in which a coalition is admissible if it consists of equal numbers of men and women, and the worth of each such coalition is the maximal total expected utility it can achieve by forming matches among themselves. ${ }^{5}$ We show that for each ex-post stable assignment, there is a utility profile such that the assignment belongs to the core of the associated TU-game (Theorem 2). That is, even if we were to allow monetary transfers, there would be no profitable ex-ante group deviation.

Our other result pertains to efficiency. A natural efficiency requirement for probabilistic assignments is sd-efficiency, which is based on the first-order stochastic dominance relation. A probabilistic assignment stochastically dominates (sd-dominates) another if for each agent, the probability distribution assigned to that agent in the former first-order stochastically dominates the probability distribution assigned to that agent in the latter assignment. Assignments that are undominated in this sense are called "sd-efficient". ${ }^{6}$ We ask the following question: Consider an ordinal preference profile and an sd-efficient assignment. Does there exist a utility profile consistent with the ordinal preferences at which the assignment is Pareto efficient? We show that for each ordinal preference profile, and for each sd-efficient assignment, one can construct a utility profile consistent with the ordinal preferences such that the sum of the expected utilities of the agents is maximized at that assignment (Theorem 1). To prove the result, we characterize the sd-efficiency of an assignment in terms of a property of an order relation that we define over the set of man-woman pairs. ${ }^{7}$

Our results regarding efficiency are intimately related to some recent results. Carroll (2011) proves a counterpart of Theorem 1 in a more general social choice setup, from which Theorem 1 can be obtained as a corollary. Aziz et al. (2015) provide an interesting non-expected utility generalization of Carroll (2011). Our results are based on an order theoretical analysis; we characterize the sd-efficiency of an assignment in terms of the acyclicity of a binary relation on the set of man-woman pairs, which parallels the results by Bogomolnaia and Moulin (2001) and Katta and Sethuraman (2006). In this vein, Aziz et al. (2015) note that it does not seem possible to extend this characterization to Carroll's (2011) or their general social choice setting. Moreover, the utility profile constructed in Theorem 1 clearly relates to the utility profile constructed in Theorem 2, which sheds further light on the relation between sd-efficiency and ex-post stability. ${ }^{8}$

Our results for stability, unlike efficiency, cannot be directly related to the recent findings on the general social choice setup. To prove Theorem 2, we observe an interesting property of ex-post stable assignments related to the lattice structure of stable deterministic assignments (see Knuth, 1976, pp. 92-93, who attributes the discovery of this lattice structure to J.H. Conway). In Proposition 3, we show that each ex-post stable probabilistic assignment can be decomposed into a collection of deterministic stable assignments, which can be ordered in such a way that each man's welfare is non-increasing and each woman's welfare is non-decreasing as we follow the assignments from the first to the last. This result, which we show via the rounding approach due to Teo and Sethuraman (1998), plays a key role in the proof of Theorem 2. A corollary of Theorem 2 is that ex-post stability implies sd-efficiency when preferences are strict, which is independently shown also by Manjunath (2011).

\footnotetext{
2 One justification for why such mechanisms are common is that it is a complex process for an agent to formulate his utility information. See Bogomolnaia and Moulin (2001) for a detailed discussion.

3 After the realization of a deterministic assignment.

4 That is, before the realization of a particular deterministic assignment.

5 We assume preferences to be quasilinear in money.

6 This notion is usually referred to as "ordinal efficiency", starting from Bogomolnaia and Moulin (2001). Here, we use the terminology of Thomson (2010).

7 For the problem of assigning objects, similar characterizations are given by Bogomolnaia and Moulin (2001) in the case of strict preferences and by Katta and Sethuraman (2006) in the case of weak preferences.

8 In fact, as noted in the proof of Proposition 2, if the ex-post stable assignment has a decomposition into stable assignments such that no agent matches with the same agent in two different assignments, then the two utility profiles coincide.
} 


\subsection{Related literature}

For the probabilistic assignment setup, a strand of the literature discusses different stability notions and their relation to each other. At the heart of this literature lies the notion of ex-post stability, which is also at the center of our study. Roth et al. (1993), Rothblum (1992), and Vande Vate (1989) provide a characterization of the set of ex-post stable assignments that we summarize in Lemma 2 and use in the proof of Proposition 3. More recently, Manjunath (2011) investigates several ex-ante stability and core notions that are based on first-order stochastic dominance. Kesten and Ünver (2015) propose ex-ante stability notions for the school choice problem, in which schools have priority orderings rather then preferences, which are not taken into consideration in the welfare analysis. Second, schools are assumed to behave non-strategically. In all these studies, the focus is on stability and core notions based on ordinal preference information. In contrast, we are interested in the relationship between ex-post stability based on ordinal preferences and a core notion based on cardinal preferences.

Following Bogomolnaia and Moulin (2001), one strand of the literature studies the probabilistic assignment of objects. In this literature, a result similar to our Theorem 1, which is due to McLennan (2002), ${ }^{9}$ follows from our Theorem 1 (Corollary 1). Manea (2008) gives a constructive proof of McLennan's result. Our proof technique is similar to Manea's: An order relation, the acyclicity of which characterizes an assignment's sd-efficiency, is used to construct the utility profile. As shown in Manea (2008), if only one side of the market has preferences over the other, then the relation rather directly delivers the desired utility profile. However, in marriage problems, the preferences of both men and women must be taken into account, both in sd-efficiency and utilitarian social welfare considerations. We show that doing so requires a rather novel construction of the utility profile compared to the case of one-sided markets.

In the deterministic assignment literature, the theory of stable assignments without transfers (NTU-model) is first developed by Gale and Shapley (1962). Given preference rankings, the algorithm they propose selects a stable assignment that is Pareto dominant for the proposing side among all stable assignments. On the other hand, the theory of stable assignments with transfers (TU-model) is developed by Shapley and Shubik (1971), where for given valuations of agents, the core of the associated TU-game (the assignment game) is characterized. ${ }^{10}$

Our Theorem 2 offers a connection between TU and NTU-models. Echenique (2008) and Echenique et al. (2013) offer a similar connection between TU and NTU-models based on the observable content of stability in both setups. Their analysis is in a revealed preference framework, in which ordinal preferences are not observable and are recovered from an observed aggregate matching. ${ }^{11}$ Echenique et al. (2013) argue that in this setting, matching theory with transfers is nested in matching theory without transfers, that is, for each aggregate matching, if there is a utility profile such that the matching is in the core of the associated TU-game, then there is an ordinal preference profile such that the matching is the unique stable matching. For our Theorem 2, we assume that ordinal preferences and an ex-post stable assignment are observable, but utility profiles are not. We construct a utility profile that is consistent with the ordinal preferences such that the matching is in the core of the associated TU-game. Since the converse statement does not hold, ${ }^{12}$ by contrast with Echenique (2008), in our setting matching theory without transfers is nested in matching theory with transfers.

Another recent study, which provides several interesting insights on the relationship between stability in TU and NTUmodels, is Echenique and Galichon (2014). One of their goals is to understand for which stable deterministic assignments, availability of monetary transfers would not affect stability. They show that, for a particular subset of stable deterministic assignments, which they call "isolated" assignments, for any ordinal preference profile, one can construct a utility profile such that each isolated stable deterministic assignment remains stable when monetary transfers are introduced. At the end of Section 3.3, we show that their result follows from our Theorem 2.

\section{The framework}

Let $M$ be a set of $n$ men and $W$ a set of $n$ women. Each $i \in M$ has preferences over $W$, and each $j \in W$ has preferences over $M$. Let $N=M \cup W$. For each $i \in N$, the preferences of $i$, which we denote by $R_{i}$, is a weak order, that is, $R_{i}$ is transitive and complete. Let $P_{i}$ denote the associated strict preference relation, and $I_{i}$ the associated indifference relation. Let $\mathcal{R}_{i}$ denote the set of all possible preference relations for $i$, and $\mathcal{R} \equiv \times_{i \in N} \mathcal{R}_{i}$ denote the set of all possible preference profiles.

A deterministic assignment is a one-to-one function $\mu: M \cup W \rightarrow M \cup W$ such that for each $(m, w) \in M \times W$, we have $\mu(m) \in W, \mu(w) \in M$, and $\mu(m)=w$ if and only if $\mu(w)=m$. A deterministic assignment can be represented by an $n \times n$ matrix, with rows indexed by men and columns indexed by women, and having entries in $\{0,1\}$, such that each row and each column has exactly one 1 . Such a matrix is called a permutation matrix. For each $(m, w) \in M \times W$, having 1 in the $(m, w)$ entry indicates that $m$ is assigned to $w$. A probabilistic assignment is a probability distribution over deterministic assignments. A probabilistic assignment can be represented by an $n \times n$ matrix having entries in [0,1] such that the sum

\footnotetext{
9 Athanassoglou (2011) approaches the problem in McLennan (2002) by using duality.

10 This theory is applied to the marriage problem by Becker (1973).

11 See Echenique et al. (2013) for the definition of an aggregate matching.

12 See our Example 2.
} 
of the entries in each row and each column is 1 . Such a matrix is a doubly stochastic matrix. For each probabilistic assignment $\pi$, and each pair $(m, w) \in M \times W$, the entry $\pi_{m w}$ indicates the probability that $m$ is assigned to $w$ at $\pi$. Since each doubly stochastic matrix can be represented as a convex combination of permutation matrices (Birkhoff, 1946 and Von Neumann, 1953), the set of all doubly stochastic matrices provides another representation for the set of all probabilistic assignments. Let $\Pi$ be the set of all doubly stochastic matrices.

We denote the collection of all lotteries over $M$ by $\mathcal{L}(M)$, and the collection of all lotteries over $W$ by $\mathcal{L}(W)$. For each $i \in M$ with preferences $R_{i} \in \mathcal{R}_{i}$, a von Neumann-Morgenstern (vNM) utility function $u_{i}$ is a real-valued mapping on $W$, i.e. $\boldsymbol{u}_{\boldsymbol{i}}: \boldsymbol{W} \rightarrow \mathbb{R}$. We obtain the corresponding preferences of $i$ over $\mathcal{L}(W)$ by comparing expected utilities. For each $i \in M$ with preferences $R_{i} \in \mathcal{R}_{i}$, a vNM utility function $u_{i}$ is consistent with $\boldsymbol{R}_{\boldsymbol{i}}$ if for each pair $\left(w, w^{\prime}\right) \in W$ we have $u_{i}(w) \geq u_{i}\left(w^{\prime}\right)$ if and only if $w R_{i} w^{\prime}$. For each woman, a (vNM) utility function consistent with her ordinal preferences is defined similarly.

For each utility profile $u=\left(u_{i}\right)_{i \in N}$ and probabilistic assignment $\pi$, the utilitarian social welfare at $(\boldsymbol{u}, \boldsymbol{\pi})$ is the sum of the expected utilities of the agents, that is:

$$
S W(u, \pi)=\sum_{(m, w) \in M \times W} \pi_{m w}\left(u_{m}(w)+u_{w}(m)\right) .
$$

An assignment $\boldsymbol{\pi}$ is ex-ante utilitarian-welfare maximizing at a utility profile $\boldsymbol{u}$ if it maximizes the social welfare at $u$, i.e. $\pi \in \operatorname{argmax}_{\pi \in \Pi} S W(u, \pi)$.

Next, we define a well-known notion of efficiency that is independent of any vNM utility specification consistent with the ordinal preferences. Let $\pi, \pi^{\prime} \in \Pi$ and $R \in \mathcal{R}$; we say that $\boldsymbol{\pi}$ first-order stochastically dominates $\boldsymbol{\pi}^{\prime}$ at $\boldsymbol{R}$ if for each pair $(m, w) \in M \times W$ :

$$
\sum_{w^{\prime}: w^{\prime} R_{m} w} \pi_{m w^{\prime}} \geq \sum_{w^{\prime}: w^{\prime} R_{m} w} \pi_{m w^{\prime}}^{\prime} \text { and } \sum_{m^{\prime}: m^{\prime} R_{w} m} \pi_{m^{\prime} w} \geq \sum_{m^{\prime}: m^{\prime} R_{w} m} \pi_{m^{\prime} w}^{\prime}
$$

such that for at least one pair, at least one of the inequalities is strict. An assignment $\boldsymbol{\pi} \in \boldsymbol{\Pi}$ is sd-efficient at $\boldsymbol{R}$ if no probabilistic assignment sd-dominates $\pi$ at $R$. For each $R \in \mathcal{R}$, let $P^{s d}(R)$ denote the set of sd-efficient assignments at $R$.

\section{Results}

\subsection{A characterization of sd-efficiency}

For each pair $(\pi, R) \in \Pi \times \mathcal{R}$, we define two relations $\sim_{(\pi, R)}$ and $\succ_{(\pi, R)}$ on $M \times W$ induced by $(\pi, R)$, and characterize the sd-efficiency of an assignment $\pi$ at $R$ in terms of a property of $\sim_{(\pi, R)}$ and $\succ(\pi, R)$.

For each pair $(m, w),\left(m^{\prime}, w^{\prime}\right) \in M \times W,(m, w) \sim_{(\pi, R)}\left(m^{\prime}, w^{\prime}\right)$ if and only if $\pi_{m w}>0, \pi_{m^{\prime}} w^{\prime}>0$, and $w^{\prime} I_{m} w, m I_{w^{\prime}} m^{\prime}$. For each pair $(m, w),\left(m^{\prime}, w^{\prime}\right) \in M \times W,(m, w) \succ_{(\pi, R)}\left(m^{\prime}, w^{\prime}\right)$ if and only if $\pi_{m, w}>0, \pi_{m^{\prime} w^{\prime}}>0$, and $w^{\prime} R_{m} w, m R_{w^{\prime}}$ $m^{\prime}$ with at least one strict preference. Let the relation $\succeq_{(\pi, R)}$ be the union of the two relations just defined. That is $\succeq_{(\pi, R)}=\sim_{(\pi, R)} \cup \succ_{(\pi, R)}$.

If assignment $\pi$ is deterministic and preferences are strict, then $(m, w) \succ_{(\pi, R)}\left(m^{\prime}, w^{\prime}\right)$ implies that $m$ and $w^{\prime}$ prefer each other to their assigned mates. Furthermore, suppose that $(m, w) \succ_{(\pi, R)}\left(m^{\prime}, w^{\prime}\right) \succ_{(\pi, R)}(m, w)$. Then, $m$ and $m^{\prime}$ (or $w$ and $w^{\prime}$ ) are better off by exchanging mates. In general, if the relation $\succ_{(\pi, R)}$ has a cycle, agents in the cycle can Pareto improve by exchanging mates along the cycle. Next, we formulate an acyclicity requirement on $\succeq_{(\pi, R)}$ with the same implication for sd-efficiency.

Note that, if $(m, w) \succ_{(\pi, R)}\left(m^{\prime}, w^{\prime}\right)$, then $(m, w)$ and $\left(m^{\prime}, w^{\prime}\right)$ are not related according to $\sim_{(\pi, R)}$, that is, $(m, w) \nsim_{(\pi, R)}$ $\left(m^{\prime}, w^{\prime}\right)$. A strong cycle of $\succeq_{(\pi, R)}$ is a sequence of pairs $\left(m_{1}, w_{1}\right),\left(m_{2}, w_{2}\right), \ldots,\left(m_{k}, w_{k}\right) \in M \times W$ such that $\left(m_{1}, w_{1}\right) \succ(\pi, R)$ $\left(m_{2}, w_{2}\right) \succeq_{(\pi, R)} \ldots \succeq_{(\pi, R)}\left(m_{k}, w_{k}\right) \succeq_{(\pi, R)}\left(m_{1}, w_{1}\right)$. The relation $\succeq_{(\pi, \boldsymbol{R})}$ is weakly acyclic if and only if it has no strong cycle. Next, we characterize sd-efficient assignments. This result generalizes characterizations of sd-efficiency of object assignments on the strict preference domain (Bogomolnaia and Moulin, 2001) and on the weak preference domain (Katta and Sethuraman, 2006).

Proposition 1. An assignment $\pi$ is sd-efficient at a preference profile $R$ if and only if $\succeq_{(\pi, R)}$ is weakly acyclic.

Proof. Let $\pi \in \Pi, R \in \mathcal{R}$.

Only-if part: We prove the contrapositive statement. Suppose that $\succeq_{(\pi, R)}$ is not weakly acyclic, that is, there is a sequence of pairs $\left(m_{1}, w_{1}\right),\left(m_{2}, w_{2}\right), \ldots,\left(m_{k}, w_{k}\right) \in M \times W$ such that $\left(m_{1}, w_{1}\right) \succ_{(\pi, R)}\left(m_{2}, w_{2}\right) \succeq_{(\pi, R)} \ldots \succeq_{(\pi, R)}\left(m_{k}, w_{k}\right) \succeq_{(\pi, R)}$ $\left(m_{1}, w_{1}\right)$. Let $\epsilon \equiv \min _{i \in\{1, \ldots, k\}} \pi_{m_{i} w_{i}}$. Let $\pi^{\prime} \in \Pi$ be defined by setting for each $i \in\{1, \ldots, k\}, \pi_{m_{i} w_{i}}^{\prime}=\pi_{m_{i} w_{i}}-\epsilon, \pi_{m_{i} w_{i+1}}^{\prime}=$ $\pi_{m_{i} w_{i+1}}+\epsilon$ (with the convention that $w_{k+1}=w_{1}$ ), and for each other pair $(m, w), \pi_{m w}^{\prime}=\pi_{m w}$. Note that $\pi^{\prime}$ sd-dominates $\pi$ at $R$. Thus, $\pi \in P^{s d}(R)$.

If part: We prove the contrapositive statement. Suppose that $\pi \notin P^{s d}(R)$, that is, there is $\pi^{\prime} \in \Pi$ that sd-dominates it. Without loss of generality, suppose that there is a man, say $m_{1} \in M$, who is better off at $\pi^{\prime}$ in stochastic dominance terms. Then, there are $w_{1}, w_{2} \in W$ such that $w_{2} P_{m_{1}} w_{1}, \pi_{m_{1} w_{2}}^{\prime}>\pi_{m_{1} w_{2}}$, and $\pi_{m_{1} w_{1}}^{\prime}<\pi_{m_{1} w_{1}}$. Moreover, there is $m_{2} \in M$ 
such that $m_{1} R_{w_{2}} m_{2}$ and $\pi_{m_{2} w_{2}}^{\prime}<\pi_{m_{2} w_{2}}$. Note that $\left(m_{1}, w_{1}\right) \succ_{(\pi, R)}\left(m_{2}, w_{2}\right)$. Now, there are $m_{3} \in M, w_{3} \in W$ such that $w_{3} R_{m_{2}} w_{2}, \pi_{m_{2} w_{3}}^{\prime}>\pi_{m_{2} w_{3}}, m_{2} R_{w_{3}} m_{3}$, and $\pi_{m_{3} w_{3}}^{\prime}<\pi_{m_{3} w_{3}}$. Then, $\left(m_{1}, w_{1}\right) \succ_{(\pi, R)}\left(m_{2}, w_{2}\right) \succeq_{(\pi, R)}\left(m_{3}, w_{3}\right)$. Proceeding inductively, we can add pairs to this sequence, and since there are finitely many man-woman pairs, this sequence includes a cycle of $\succeq(\pi, R)$.

If the cycle includes $\left(m_{1}, w_{1}\right)$, then it is strong and we are done. Suppose otherwise. Let the cycle consist of $\left(x_{1}, y_{1}\right),\left(x_{2}, y_{2}\right), \ldots,\left(x_{k}, y_{k}\right) \in M \times W$. Note that $\left(x_{1}, y_{1}\right) \sim_{(\pi, R)}\left(x_{2}, y_{2}\right) \sim_{(\pi, R)} \ldots \sim_{(\pi, R)}\left(x_{k}, y_{k}\right) \sim_{(\pi, R)}\left(x_{1}, y_{1}\right)$. Remember that $\pi_{x_{1} y_{1}}^{\prime}<\pi_{x_{1} y_{1}}, \pi_{x_{2} y_{2}}^{\prime}<\pi_{x_{2} y_{2}}, \ldots, \pi_{x_{k} y_{k}}^{\prime}<\pi_{x_{k} y_{k}}$, and $\pi_{x_{1} y_{2}}^{\prime}>\pi_{x_{1} y_{2}}, \pi_{x_{2} y_{3}}^{\prime}>\pi_{x_{2} y_{3}}, \ldots, \pi_{x_{k} y_{1}}^{\prime}>\pi_{x_{k} y_{1}}$. Let $\epsilon_{1} \equiv$ $\min \left\{\pi_{x_{1} y_{1}}-\pi_{x_{1} y_{1}}^{\prime}, \ldots, \pi_{x_{k} y_{k}}-\pi_{x_{k} y_{k}}^{\prime}\right\}, \epsilon_{2} \equiv \min \left\{\pi_{x_{1} y_{2}}^{\prime}-\pi_{x_{1} y_{2}}, \ldots, \pi_{x_{k} y_{1}}^{\prime}-\pi_{x_{k} y_{1}}\right\}$ and $\epsilon \equiv \min \left\{\epsilon_{1}, \epsilon_{2}\right\}$. Let $\pi^{\prime \prime} \in \Pi$ be defined by setting for each $i \in\{1, \ldots, k\}, \pi_{x_{i} y_{i}}^{\prime \prime}=\pi_{x_{i} y_{i}}^{\prime}+\epsilon, \pi_{x_{i} y_{i+1}}^{\prime \prime}=\pi_{x_{i} y_{i+1}}^{\prime}-\epsilon$ (with the convention that $y_{k+1}=y_{1}$ ), and for each other pair $(m, w), \pi_{m w}^{\prime \prime}=\pi_{m w}^{\prime}$. Since for each $i \in\{1, \ldots, k\}, y_{i} I_{x_{i}} y_{i+1}$ and $x_{i-1} I_{y_{i}} x_{i}$, $\pi^{\prime \prime}$ also sd-dominates $\pi$. Also, by the construction of $\pi^{\prime \prime}$, there are two consecutive pairs in the cycle, say $\left(x_{t}, y_{t}\right)$ and $\left(x_{t+1}, y_{t+1}\right)$, such that either $\pi_{x_{t} y_{t}}^{\prime \prime}=\pi_{x_{t} y_{t}}$ or $\pi_{x_{t} y_{t+1}}^{\prime \prime}=\pi_{x_{t} y_{t+1}}$.

Now, because $\pi^{\prime \prime}$ sd-dominates $\pi$, as we did above, we can find a cycle of $\succeq_{(\pi, R)}$, say $\left(x_{1}^{\prime}, y_{1}^{\prime}\right),\left(x_{2}^{\prime}, y_{2}^{\prime}\right), \ldots,\left(x_{t}^{\prime}, y_{t}^{\prime}\right) \in$ $M \times W$, such that for each $i \in\{1, \ldots, t\}, \pi_{x_{i}^{\prime} y_{i}^{\prime}}^{\prime \prime}<\pi_{x_{i}^{\prime} y_{i}^{\prime}}$ and $\pi_{x_{i}^{\prime} y_{i+1}^{\prime}}^{\prime \prime}>\pi_{x_{i}^{\prime} y_{i+1}^{\prime}}$ (with the convention that $y_{t+1}=y_{1}$ ). Note that $\left(x_{t}, y_{t}\right)$ and $\left(x_{t+1}, y_{t+1}\right)$ cannot be part of this cycle consecutively, implying that this new cycle must be different from the cycle that was identified before. Continuing similarly, we obtain additional assignments that sd-dominate $\pi$ and additional cycles. None of those cycles can include $\left(x_{t}, y_{t}\right)$ and $\left(x_{t+1}, y_{t+1}\right)$ consecutively; and for each additional cycle, we identify additional consecutive pairs in the cycle that cannot be part of any cycle in the future, implying that each cycle is different from any cycle that was identified before. Since the number of cycles in $\succeq_{(\pi, R)}$ is finite, this process eventually leads to a strong cycle. Thus, $\succeq_{(\pi, R)}$ is not weakly acyclic.

Given $R \in \mathcal{R}$, for each $\pi \in P^{s d}(R)$, the relation $\succeq_{(\pi, R)}$ can have cycles of the form $\left(m_{1}, w_{1}\right) \succeq_{(\pi, R)}\left(m_{2}, w_{2}\right) \succeq_{(\pi, R)}$ $\ldots \succeq_{(\pi, R)}\left(m_{k}, w_{k}\right) \succeq_{(\pi, R)}\left(m_{1}, w_{1}\right)$. However, $\succeq_{(\pi, R)}$ being weakly acyclic implies that such a cycle should belong to $\sim_{(\pi, R)}$, that is, the cycle should be of the form $\left(m_{1}, w_{1}\right) \sim_{(\pi, R)}\left(m_{2}, w_{2}\right) \sim_{(\pi, R)} \ldots \sim_{(\pi, R)}\left(m_{k}, w_{k}\right) \sim_{(\pi, R)}\left(m_{1}, w_{1}\right)$.

Given $(\pi, R) \in \Pi \times \mathcal{R}$, let $C_{\pi, R}$ be the binary relation on $M \times W$, defined as follows: For each pair $(m, w),\left(m^{\prime}, w^{\prime}\right) \in$ $M \times W,(m, w) C_{\pi, R}\left(m^{\prime}, w^{\prime}\right)$ if and only if there is a cycle of $\sim_{(\pi, R)}$ that contains both, that is, there is a sequence of pairs (not necessarily distinct) $\left(m_{1}, w_{1}\right),\left(m_{2}, w_{2}\right), \ldots,\left(m_{k}, w_{k}\right) \in M \times W$ that includes $(m, w)$ and $\left(m^{\prime}, w^{\prime}\right)$, and is such that $\left(m_{1}, w_{1}\right) \sim_{(\pi, R)}\left(m_{2}, w_{2}\right) \sim_{(\pi, R)} \ldots \sim_{(\pi, R)}\left(m_{k}, w_{k}\right) \sim_{(\pi, R)}\left(m_{1}, w_{1}\right)$. Note that $C_{\pi, R}$ is an equivalence relation on $M \times W$, that is, it is reflexive, symmetric, and transitive. For each pair $(m, w) \in M \times W$, let $[m, w]_{C_{\pi, R}} \equiv\left\{\left(m^{\prime}, w^{\prime}\right) \in M \times W\right.$ : $\left.(m, w) C_{\pi, R}\left(m^{\prime}, w^{\prime}\right)\right\}$ denote the equivalence class of $(m, w)$ relative to $C_{\pi, R}$. Let $\gg_{(\pi, R)}$ be the relation defined on the set of all equivalence classes of $C_{(\pi, R)}$ as follows: For each pair $\left(m_{1}, w_{1}\right),\left(m_{2}, w_{2}\right) \in M \times W,\left[m_{1}, w_{1}\right] \gg(\pi, R)\left[m_{2}, w_{2}\right]$ if and only if $\left[m_{1}, w_{1}\right] \neq\left[m_{2}, w_{2}\right]$ and there are $\left(m_{1}^{\prime}, w_{1}^{\prime}\right) \in\left[m_{1}, w_{1}\right],\left(m_{2}^{\prime}, w_{2}^{\prime}\right) \in\left[m_{2}, w_{2}\right]$ such that $\left(m_{1}^{\prime}, w_{1}^{\prime}\right) \succeq_{(\pi, R)}\left(m_{2}^{\prime}, w_{2}^{\prime}\right)$. Note that, if $\pi \in P^{s d}(R)$, then $\gg_{(\pi, R)}$ is acyclic.

\subsection{An efficiency theorem}

In this section we show that for each probabilistic assignment that is sd-efficient at a given preference profile, there is a utility profile consistent with these preferences such that the probabilistic assignment maximizes the sum of the expected utilities. First, let us consider the simple case where the sd-efficient assignment for which a utility function is to be constructed is an efficient deterministic assignment, $\mu$. Let $u$ be a utility profile consistent with $R$ such that for each $(m, w) \in M \times W$, if $m$ and $w$ are matched at $\mu$, then $u_{m}(w)=u_{w}(m)=1$. Further, for each $w^{\prime} \in W$ such that $w \succ_{m} w^{\prime}$, let $0<u_{m}\left(w^{\prime}\right)<\delta$ for some $\delta>0$ and for each $w^{\prime \prime} \in W$ such that $w^{\prime \prime} \succ_{m} w$, let $1<u_{m}\left(w^{\prime \prime}\right)<1+\epsilon$ for some $\epsilon>0$. Let $u_{w}$ be similarly defined. Note that for some small enough selection of $\delta$ and $\epsilon$, the efficient assignment $\mu$ is a welfare maximizing assignment at utility profile $u$.

This construction would fail even for the simplest probabilistic assignment, which is obtained as a mixture of two efficient deterministic assignments. However, we show that the same conclusion holds for each sd-efficient probabilistic assignment $\pi$ by providing an explicit construction for a utility profile at which $\pi$ is welfare maximizing. The construction in the next example is instructive to understand the general construction to follow in Theorem 1.

Example 1. Let $M=\{1,2,3\}$ and $W=\{a, b, c\}$. Let the preference profile $R$ be as follows:

\begin{tabular}{llllll}
\hline$R_{1}$ & $R_{2}$ & $R_{3}$ & $R_{a}$ & $R_{b}$ & $R_{c}$ \\
\hline$a$ & $b$ & $c$ & 3 & 1 & 2 \\
$c$ & $a$ & $b$ & 2 & 3 & 1 \\
$b$ & $c$ & $a$ & 1 & 2 & 3 \\
\hline
\end{tabular}

Let $\mu$ be the assignment where 1 is matched with $a, 2$ with $b$, and 3 with $c$. Let $\mu^{\prime}$ be the assignment where 1 is matched with $b, 2$ with $c$, and 3 with $a$. Let $\pi$ assign 0.5 probability to each of $\mu$ and $\mu^{\prime}$. 
Now, let $u$ be such that each agent gets utility 1 from his/her top-ranked agent and utility 0 from his/her third-ranked agent. Further, each agent gets utility in the open interval $(0,1 / 2)$ from his/her second-ranked agent. Clearly, for each pair $(m, w)$, if $\pi_{m w}>0$, then $u_{m}(w)+u_{w}(m)=1$. Moreover, if $\pi_{m w}=0$, then $u_{m}(w)+u_{w}(m)<1$. Hence, the sum of expected utilities, which is 3 at $\pi$, cannot exceed 3 at any probabilistic assignment.

In the above construction, for each man-woman pair, the sum of the utilities they get from each other is the same, and that aspect plays the critical role. For our general result, a similar construction works.

Theorem 1. For each preference profile and each assignment that is sd-efficient at it, there is a utility profile consistent with the preference profile such that the assignment is ex-ante utilitarian welfare maximizing at that utility profile.

Proof. Let $(\pi, R) \in \Pi \times \mathcal{R}$ be such that $\pi \in P^{s d}(R)$. Since we fix $(\pi, R)$ throughout the proof, we remove the reference to $(\pi, R)$ in denoting the binary relations we have defined, and simply write $\sim, \succ, \succeq$, and $\gg$.

For each pair $m \in M, w \in W$, let $s_{m w}$ denote the length of the longest path of $\gg$ starting at $[m, w]$, and let $e_{m w}$ denote the length of the longest path of $\gg$ ending at $[m, w] .{ }^{13}$

Step 1: Constructing an auxiliary utility profile. For each $m \in M$, let $v_{m}: W \rightarrow \mathbb{R}$ be defined by setting, for each $w \in W$,

$$
v_{m}(w)=\frac{e_{m w}}{e_{m w}+s_{m w}} .
$$

For each $w \in W$, let $v_{w}: M \rightarrow \mathbb{R}$ be defined by setting, for each $m \in M$,

$$
v_{w}(m)=\frac{s_{m w}}{e_{m w}+s_{m w}} .
$$

Note that for each pair $(m, w) \in M \times W, v_{m}(w)+v_{w}(m)=1$.

Lemma 1. Let $(m, w),\left(m^{\prime}, w^{\prime}\right) \in M \times W$. If $[m, w] \gg\left[m^{\prime}, w^{\prime}\right]$, then $v_{w}(m)>v_{w^{\prime}}\left(m^{\prime}\right)$.

Proof. Since $e_{m w}<e_{m^{\prime} w^{\prime}}$ and $s_{m w}>s_{m^{\prime} w^{\prime}}$, then

$$
\frac{s_{m w}}{s_{m w}+e_{m w}}>\frac{s_{m^{\prime} w^{\prime}}}{s_{m^{\prime} w^{\prime}}+e_{m^{\prime} w^{\prime}}} \text {, i.e. } \frac{1}{1+\frac{e_{m w}}{s_{m w}}}>\frac{1}{1+\frac{e_{m^{\prime} w^{\prime}}}{s_{m^{\prime} w^{\prime}}}} .
$$

One consequence of the Lemma is that, for each pair $(m, w),\left(m^{\prime}, w^{\prime}\right) \in M \times W$, if $[m, w] \gg\left[m^{\prime}, w^{\prime}\right]$, then $v_{m}(w)+$ $v_{w^{\prime}}\left(m^{\prime}\right)<1$. Let $z\left((m, w),\left(m^{\prime}, w^{\prime}\right)\right)=1-v_{m}(w)-v_{w^{\prime}}\left(m^{\prime}\right)$ if $[m, w] \gg\left[m^{\prime}, w^{\prime}\right]$, and 1 otherwise. Let

$$
\min _{(m, w),\left(m^{\prime}, w^{\prime}\right) \in M \times W} z\left((m, w),\left(m^{\prime}, w^{\prime}\right)\right) \equiv 2 \epsilon .
$$

Step 2: Defining the utility profile $\boldsymbol{u}$. Let $m \in M$. Let $u_{m}$ be defined as follows (for each $w \in W$, we define $u_{w}$ in a symmetric way):

$i$. For each $w \in W$ such that $\pi_{m w}>0$, set $u_{m}(w) \equiv v_{m}(w)$. We show that $u_{m}$ is consistent with $R_{m}$ on the subset of women for whom $\pi_{m w}>0$. Let $m \in M$ and $w, w^{\prime} \in W$ be such that $\pi_{m w}>0$ and $\pi_{m w^{\prime}}>0$. Without loss of generality, suppose that $w R_{m} w^{\prime}$. If $w I_{m} w^{\prime}$, then note that $\left(m, w^{\prime}\right) \sim(m, w)$, and $\left[m, w^{\prime}\right] \sim[m, w]$. Thus, $u_{m}(w)=u_{m}\left(w^{\prime}\right)$, as desired. If $w P_{m} w^{\prime}$, then $\left(m, w^{\prime}\right) \succ(m, w)$, and $\left[m, w^{\prime}\right] \gg[m, w]$. Thus, $u_{m}(w)>u_{m}\left(w^{\prime}\right)$, as desired.

ii. For each $w \in W$ such that $\pi_{m w}=0$ and there is no $w^{\prime} \in W$ with $\pi_{m} w^{\prime}>0, w R_{m} w^{\prime}$, set $u_{m}(w) \leq-1$. Obviously, at this step the utilities can be chosen such that $u_{m}$ is consistent with $R_{m}$ on the subset of women for whom the utilities are defined so far.

iii. For each $w \in W$ such that $\pi_{m w}=0$ and there is $w^{\prime} \in W$ with $\pi_{m w^{\prime}}>0, w R_{m} w^{\prime}$, consider a best such $w^{\prime}$, that is, $\pi_{m w^{\prime}}>0, w R_{m} w^{\prime}$, and there is no such $w^{\prime \prime} \in W$ with $w^{\prime \prime} P_{m} w^{\prime}$. Set $u_{m}(w) \in\left[v_{m}\left(w^{\prime}\right), v_{m}\left(w^{\prime}\right)+\epsilon\right]$. Obviously, at this step the utilities can be chosen such that $u_{m}$ is consistent with $R_{m}$ on the entire set of women.

Let the function $S W(u,):. \Pi \rightarrow \mathbb{R}$ be defined by setting, for each $\pi^{\prime} \in \Pi$,

$$
S W\left(u, \pi^{\prime}\right)=\sum_{(m, w) \in M \times W}\left[\pi_{m w}^{\prime}\left(u_{m}(w)+u_{w}(m)\right)\right] .
$$

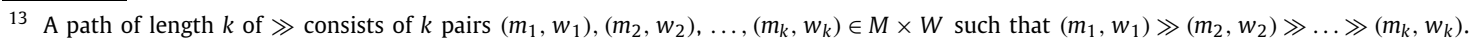


Step 3: $\boldsymbol{S} \boldsymbol{W}$ attains its maximum at $\boldsymbol{\pi}$. For each pair $(m, w) \in M \times W$, if $\pi_{m w}>0$, then $u_{m}(w)+u_{w}(m)=1$. Thus, $S W(u, \pi)=n$. We show that for each pair $(m, w) \in M \times W$, if $\pi_{m w}=0$, then $u_{m}(w)+u_{w}(m) \leq 1$, which implies that the maximal possible ex-ante utilitarian social welfare is $n$, and it is reached at $\pi$.

Let $(m, w) \in M \times W$ be such that $\pi_{m w}=0$. Suppose that there is no $w^{\prime} \in W$ such that $\pi_{m w^{\prime}}>0, w R_{m} w^{\prime}$. Then, $u_{m}(w) \leq-1$. If there is no $m^{\prime} \in M$ such that $\pi_{m^{\prime} w}>0$ and $m R_{w} m^{\prime}$, then $u_{w}(m) \leq-1$ and $u_{m}(w)+u_{w}(m)<1$. If there is $m^{\prime} \in M$ such that $\pi_{m^{\prime} w}>0$ and $m R_{w} m^{\prime}$, then $u_{w}(m) \leq u_{w}\left(m^{\prime}\right)+\epsilon<2$. Thus, $u_{m}(w)+u_{w}(m)<1$. The case when there is no $m^{\prime} \in M$ such that $\pi_{m^{\prime} w}>0, m R_{w} m^{\prime}$, is symmetric.

So, there is only one case left to consider. Suppose that there is $w^{\prime} \in W$ such that $\pi_{m w^{\prime}}>0$ and $w R_{m} w^{\prime}$, and there is $m^{\prime} \in M$ such that $\pi_{m^{\prime} w}>0$ and $m R_{w} m^{\prime}$. Let $w^{\prime}$ and $m^{\prime}$ be the best such agents. Note that $\left(m, w^{\prime}\right) \succeq\left(m^{\prime}, w\right)$. If $w I_{m} w^{\prime}$ and $m I_{w} m^{\prime}$, then by Lemma $1, u_{m}(w)+u_{w}(m)<1$. So, suppose that for at least one agent, the preference is strict. Then, $\left(m, w^{\prime}\right) \succ\left(m^{\prime}, w\right)$ and $\left[m, w^{\prime}\right] \gg\left[m^{\prime}, w\right]$. Recall that $z\left(\left(m, w^{\prime}\right),\left(m^{\prime}, w\right)\right)=1-v_{m}\left(w^{\prime}\right)-v_{w}\left(m^{\prime}\right) \geq 2 \epsilon$. Now,

$$
u_{m}(w)+u_{w}(m) \leq\left[u_{m}\left(w^{\prime}\right)+\epsilon\right]+\left[u_{w}\left(m^{\prime}\right)+\epsilon\right]=v_{m}\left(w^{\prime}\right)+v_{w}\left(m^{\prime}\right)+2 \epsilon
$$

which implies $u_{m}(w)+u_{w}(m) \leq 1$, as desired.

The welfare theorem by McLennan (2002) for the problem of allocating objects is a corollary of Theorem 1 . To see this, first consider the following counterparts of sd-efficiency and utilitarian social welfare for that model. Let us keep the men-women notation. An assignment $\boldsymbol{\pi} \in \boldsymbol{\Pi}$ men-side sd-dominates $\boldsymbol{\pi}^{\prime} \in \boldsymbol{\Pi}$ at $\boldsymbol{R} \in \mathcal{R}$ if for each agent $i \in M$, the lottery assigned to $i$ at $\pi$ sd-dominates the one assigned at $\pi^{\prime}$. That is, for each pair $(m, w) \in M \times W$,

$$
\sum_{w^{\prime}: w^{\prime} R_{m} w} \pi_{m w^{\prime}} \geq \sum_{w^{\prime}: w^{\prime} R_{m} w} \pi_{m w^{\prime}}^{\prime}
$$

such that for at least one pair the inequality is strict. An assignment $\boldsymbol{\pi} \in \boldsymbol{\Pi}$ is men-side sd-efficient at $\boldsymbol{R} \in \mathcal{R}$ if no probabilistic assignment men-side sd-dominates it at $R$. For each utility profile $u=\left(u_{i}\right)_{i \in N}$ and probabilistic assignment $\pi$, the men-side utilitarian social welfare at $(\boldsymbol{u}, \boldsymbol{\pi})$ is the sum of the utilities of the men, that is:

$$
\operatorname{MSW}(u, \pi)=\sum_{m \in M} \sum_{w \in W} \pi_{m w} u_{m}(w) .
$$

A probabilistic assignment $\boldsymbol{\pi}$ is ex-ante men-side utilitarian welfare maximizing at a utility profile $\boldsymbol{u}$ if it maximizes the men-side social welfare at $u$, that is, $\pi \in \operatorname{argmax}_{\pi \in \Pi} M S W(u, \pi)$.

Corollary 1. (See McLennan, 2002.) For each preference profile and each assignment that is men-side sd-efficient at it, there is a utility profile consistent with the preference profile such that the assignment is ex-ante men-side utilitarian welfare maximizing at that utility profile.

Proof. Let $\pi \in \Pi$ be men-side sd-efficient at $R \in \mathcal{R}$. Let $R^{\prime} \in \mathcal{R}$ be such that for each $m \in M, R_{m}^{\prime}=R_{m}$, and each woman is indifferent between any two men at $R^{\prime}$. Note that $\pi$ is men-side sd-efficient also at $R^{\prime}$. Moreover, $\pi \in P^{s d}\left(R^{\prime}\right)$. By Theorem 1 , there is a utility profile $u$ consistent with $R^{\prime}$ such that $\pi$ is ex-ante utilitarian welfare maximizing at $u$. Since each woman gets the same utility from any two men at $u, \pi$ is ex-ante men-side utilitarian welfare maximizing at $u$. Now, let $u^{\prime}$ be a utility profile such that for each $m \in M, u_{m}=u_{m}^{\prime}$, and for each $w \in W, u_{w}^{\prime}$ is consistent with $R_{w}$. Note that $\pi$ is ex-ante men-side utilitarian welfare maximizing at $u^{\prime}$.

\subsection{A stability theorem}

A central robustness criterion for deterministic assignments is "stability", which requires that there be no unmatched man-woman pair who prefer each other to their assigned mates. A counterpart of stability for probabilistic assignments is "ex-post" stability, which requires that there be at least one decomposition of the probabilistic assignment into stable deterministic assignments.

Let $\mathcal{D}$ denote the set of deterministic assignments. An assignment $\boldsymbol{\mu} \in \mathcal{D}$ is stable at $\boldsymbol{R} \in \mathcal{R}$ if there is no ( $m, w) \in$ $M \times W$ such that $m P_{w} \mu(w), w P_{m} \mu(m)$. An assignment $\boldsymbol{\pi} \in \boldsymbol{\Pi}$ is ex-post stable if it can be expressed as a convex combination of stable deterministic assignments.

From this point on, we restrict ourselves to strict preferences. For each $i \in N$, let $\mathcal{P}_{i} \subset \mathcal{R}_{i}$ be the set of all transitive, anti-symmetric, and complete preference relations for $i$. Let $\mathcal{P}=\times_{i \in N} \mathcal{P}_{i}$ be the set of all strict preference profiles.

We show that ex-post stability implies welfare properties, beyond sd-efficiency, which are also deducible from the ordinal preferences and can avoid ex-ante break aways of men-women coalitions from the society.

A coalition $S=M^{\prime} \cup W^{\prime} \subseteq M \cup W$ is admissible if $\left|M^{\prime}\right|=\left|W^{\prime}\right|$. Let $\mathcal{A}$ be the set of all admissible coalitions. For each $S \in \mathcal{A}$, let $\Pi^{S}$ denote the set of probabilistic assignments defined over $S$. For each utility profile $u=\left(u_{i}\right)_{i \in N}$, let $V^{u}$ be the 
transferable utility game defined by setting for each $S=M^{\prime} \cup W^{\prime} \in \mathcal{A}, V^{u}(S)$ to be the maximum total expected utility coalition $S$ can achieve among its members. That is, for each $S=M^{\prime} \cup W^{\prime} \in \mathcal{A}$,

$$
V^{u}(S)=\max _{\pi^{S} \in \Pi^{S}} \sum_{(m, w) \in M^{\prime} \times W^{\prime}} \pi_{m w}^{S}\left(u_{m}(w)+u_{w}(m)\right) .
$$

Let $E\left(u_{m} \mid \pi\right)=\sum_{w \in W} \pi_{m w} u_{m}(w)$ be the expected value of $u_{m}$ at $\pi$ and $E\left(u_{w} \mid \pi\right)=\sum_{m \in M} \pi_{m w} u_{w}(m)$ the expected value of $u_{w}$ at $\pi$. Given a utility profile $u$, an assignment $\pi \in \Pi$ is in the core of $\boldsymbol{V}^{\boldsymbol{u}}$ if no coalition can increase its total expected utility by deviating to another probabilistic assignment where they are matched among themselves. That is, for each $S \in \mathcal{A}$,

$$
V^{u}(S) \leq \sum_{m \in M^{\prime}} E\left(u_{m} \mid \pi\right)+\sum_{w \in W^{\prime}} E\left(u_{w} \mid \pi\right)
$$

Let $C\left(V^{u}\right)$ be the set of all assignments that are in the core of $\boldsymbol{V}^{\boldsymbol{u}}$.

Let $P \in \mathcal{P}$. Let $P_{M}$ and $P_{W}$ denote the common preferences of men and women over deterministic assignments induced by $P$, defined as follows: For each pair $\mu, \mu^{\prime} \in \mathcal{D}, \mu P_{M} \mu^{\prime}$ if and only if for each $m \in M, \mu R_{m} \mu^{\prime}$ with strict preference for some $m$. The relation $P_{W}$ is defined similarly.

An assignment $\pi \in \Pi$ is well-ordered ex-post stable at $\boldsymbol{P} \in \mathcal{P}$ if it has a decomposition into stable assignments $\mu_{1}, \ldots, \mu_{T}$ such that for each $t, t^{\prime} \in\{1, \ldots, T\}$ with $t<t^{\prime}$, we have $\mu_{t} P_{M} \mu_{t^{\prime}}$ and $\mu_{t^{\prime}} P_{W} \mu_{t}$.

Proposition 2. If an assignment $\pi$ is well-ordered ex-post stable at a strict preference profile, then there is a utility profile $u$ consistent with the preference profile such that $\pi \in C\left(V^{u}\right)$.

Proof. Let $\pi \in \Pi$ be well-ordered ex-post stable at $P \in \mathcal{P}$. Suppose that $\pi$ has the following decomposition into stable assignments: $\pi=\lambda_{1} \mu_{1}+\lambda_{2} \mu_{2}+\cdots+\lambda_{T} \mu_{T}$. Suppose that for each pair $t, t^{\prime} \in\{1,2, \ldots, T\}$ such that $t<t^{\prime}, \mu_{t} P_{M} \mu_{t^{\prime}}$ and $\mu_{t^{\prime}} P_{W} \mu_{t}$.

We first define the utilities each agent gets from the agents that he/she is matched with positive probability. Let $(m, w) \in$ $M \times W$ such that $\pi_{m w}>0$. First note that if $m$ is matched to $w$ in two different assignments in the decomposition, say $\mu_{t}, \mu_{t^{\prime}}, t<t^{\prime}$, then they should be matched in all assignments between $\mu_{t}$ and $\mu_{t^{\prime}}$, that is, for each $t^{\prime \prime} \in\left\{t, t+1, \ldots, t^{\prime}\right\}$ $\mu_{t^{\prime \prime}}(m)=w$. So, let $\mu_{p}, \mu_{p+1}, \ldots, \mu_{q}$ be the list of assignments at which $m$ is matched to $w$. Let $\lambda_{m w} \equiv \lambda_{p}+\lambda_{p+1}+\cdots+\lambda_{q}$ Let

and

$$
u_{w}(m)=\frac{\lambda_{p}}{\lambda_{m w}} \cdot \frac{p}{T+1}+\frac{\lambda_{p+1}}{\lambda_{m w}} \cdot \frac{p+1}{T+1}+\cdots+\frac{\lambda_{q}}{\lambda_{m w}} \cdot \frac{q}{T+1},
$$

$$
u_{m}(w)=\frac{\lambda_{p}}{\lambda_{m w}} \cdot \frac{T-p+1}{T+1}+\frac{\lambda_{p+1}}{\lambda_{m w}} \cdot \frac{T-p}{T+1}+\cdots+\frac{\lambda_{q}}{\lambda_{m w}} \cdot \frac{T-q+1}{T+1} .
$$

If there is a unique assignment $\mu_{t}$ in the decomposition such that $\mu_{t}(m)=w$, we simply have $u_{w}(m)=\frac{t}{T+1}$ and $u_{m}(w)=$ $\frac{T-t+1}{T+1} \cdot 14$

Next, we argue that the utilities each agent gets from the agents that he/she is matched with zero probability can be defined in such a way that for each such pair $(m, w) \in M \times W, u_{m}(w)+u_{w}(m)<1$. Let $(m, w) \in M \times W$ be such that $\pi_{m w}=0$. If there is no $w^{\prime} \in W$ such that $\pi_{m w^{\prime}}>0$ and $w P_{m} w^{\prime}$, then let $u_{m}(w) \leq-1$. The case when there is no $m^{\prime} \in W$ such that $\pi_{m^{\prime} w}>0, m P_{w} m^{\prime}$ is the same. So, suppose that there are $m^{\prime} \in M, w^{\prime} \in W$ such that $\pi_{m w^{\prime}}>0, w P_{m} w^{\prime}$, and $\pi_{m^{\prime} w}>0, m P_{w} m^{\prime}$. Suppose w.l.o.g. that $w^{\prime}$ and $m^{\prime}$ are best such agents at $P_{w}$ and $P_{m}$. We will show that $u_{m}\left(w^{\prime}\right)+$ $u_{w}\left(m^{\prime}\right)<1$. First, note that the pairs $\left(m, w^{\prime}\right)$ and $\left(m^{\prime}, w\right)$ cannot appear in the same assignment of the decomposition, since otherwise that assignment would not be stable. Suppose that $m$ and $w^{\prime}$ are matched in assignments $\mu_{p}, \mu_{p+1}, \ldots, \mu_{q}$, and $m^{\prime}$ and $w$ are matched in assignments $\mu_{p^{\prime}}, \mu_{p^{\prime}+1}, \ldots, \mu_{q^{\prime}}$. Either $q<p^{\prime}$ or $q^{\prime}<p$. In fact we cannot have $q<p^{\prime}$; otherwise, $m$ would prefer his mate in $\mu_{q}$, namely $w^{\prime}$, to his mate in $\mu_{p^{\prime}}$. But then, $m$ would prefer $w$ to his mate in $\mu_{p^{\prime}}$, contradicting the assumption that $\mu_{p^{\prime}}$ is stable. Thus $q^{\prime}<p$. Since $u_{m}\left(w^{\prime}\right) \leq \frac{T-p+1}{T+1}$ and $u_{w}\left(m^{\prime}\right) \leq \frac{q^{\prime}}{T+1}, u_{m}\left(w^{\prime}\right)+u_{w}\left(m^{\prime}\right)<1$. Then by arguments similar to the proof of Theorem 1 , for each such $m, w, m^{\prime}, w^{\prime}$, let $z\left((m, w),\left(m^{\prime}, w^{\prime}\right)\right)=1-u_{m}(w)-u_{w^{\prime}}\left(m^{\prime}\right)$ and let $2 \epsilon$ be the minimum of $z\left((m, w),\left(m^{\prime}, w^{\prime}\right)\right)$. Now, for the pair $(m, w)$, let $u_{m}(w) \in\left[u_{m}\left(w^{\prime}\right), u_{m}\left(w^{\prime}\right)+\epsilon\right]$ and let $u_{w}(m) \in\left[u_{w}\left(m^{\prime}\right), u_{w}\left(m^{\prime}\right)+\epsilon\right]$. Thus, utility profile $u$ is consistent with $P$ and for each pair $(m, w)$ that is matched with zero probability $u_{m}(w)+u_{w}(m)<1$.

Now, we show that $\pi \in C\left(V^{u}\right)$. First, for each admissible coalition $S=M^{\prime} \cup W^{\prime}, V^{u}(S) \leq\left|M^{\prime}\right|$. Now, let $(m, w) \in M \times W$. We show that $E\left(u_{m} \mid \pi\right)+E\left(u_{w} \mid \pi\right)=1$. Observe that

$$
E\left(u_{m} \mid \pi\right)=\lambda_{1} \frac{T}{T+1}+\lambda_{2} \frac{T-1}{T+1}+\cdots+\lambda_{T} \frac{1}{T+1} .
$$

\footnotetext{
14 In this case, the utility function is exactly the one constructed in Theorem 1.
} 
Similarly,

$$
E\left(u_{w} \mid \pi\right)=\lambda_{1} \frac{1}{T+1}+\lambda_{2} \frac{2}{T+1}+\cdots+\lambda_{T} \frac{T}{T+1} .
$$

It follows that $E\left(u_{m} \mid \pi\right)+E\left(u_{w} \mid \pi\right)=\lambda_{1}+\lambda_{2}+\cdots+\lambda_{T}=1$, and for each admissible coalition $S=M^{\prime} \cup W^{\prime}$,

$$
\sum_{i \in S} E\left(u_{i} \mid \pi\right)=\left|M^{\prime}\right| \geq V^{u}(S) .
$$

Therefore, $\pi \in C\left(V^{u}\right)$.

Next, we show that well-ordered ex-post stability is equivalent to ex-post stability. The proof relies on the following lemma. The first part of the lemma is due to Rothblum (1992) and the second part to Roth et al. (1993).

Lemma 2. Let $P \in \mathcal{P}$ and $\pi \in \Pi$. Let $\pi$ be ex-post stable at $P$.

1. For each pair $(m, w) \in M \times W$,

$$
\pi_{m w}+\sum_{w^{\prime}: w P_{m} w^{\prime}} \pi_{m w^{\prime}}+\sum_{m^{\prime}: m P_{w} m^{\prime}} \pi_{m^{\prime} w} \leq 1 .
$$

2. For each pair $(m, w) \in M \times W$ such that $\pi_{m w}>0$,

$$
\pi_{m w}+\sum_{w^{\prime}: w P_{m} w^{\prime}} \pi_{m w^{\prime}}+\sum_{m^{\prime}: m P_{w} m^{\prime}} \pi_{m^{\prime} w}=1 .
$$

Proposition 3. If an assignment is ex-post stable at a strict preference profile, then it has a well-ordered stable decomposition.

Proof. Let $\pi \in \Pi$ be ex-post stable at $P \in \mathcal{P}$. We construct a well-ordered stable decomposition of $\pi$ using the rounding approach due to Teo and Sethuraman (1998).

For each $m \in M$, let $\pi_{m}^{+} \equiv\left\{w \in W: \pi_{m w}>0\right\}$. We partition the $(0,1]$ interval into $\left|\pi_{m}^{+}\right|$intervals $\left(I_{m w}\right)_{w \in \pi_{m}^{+}}$such that each interval $I_{m w}$ is of the form ( $a_{m w}, b_{m w}$ ] (left-open, right-closed) with length $\pi_{m w}$, and if $w P_{m} w^{\prime}$, then $a_{m w}<a_{m w^{\prime}}$.

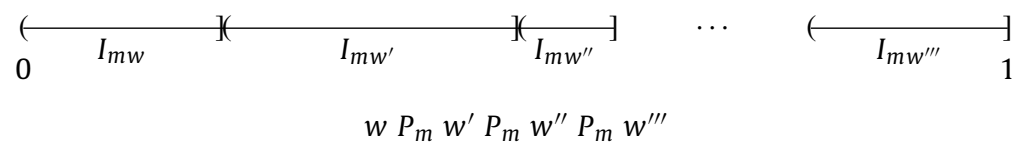

For each $w \in W$, let $\pi_{w}^{+} \equiv\left\{m \in M: \pi_{m w}>0\right\}$. We partition the $(0,1]$ interval into $\left|\pi_{w}^{+}\right|$intervals $\left(I_{w m}\right)_{m \in \pi_{w}^{+}}$such that each interval $I_{w m}$ is of the form ( $a_{w m}, b_{w m}$ ] (left-open, right-closed) with length $\pi_{m w}$, and if $m P_{w} m^{\prime}$, then $a_{w m}>a_{w m^{\prime}}$.

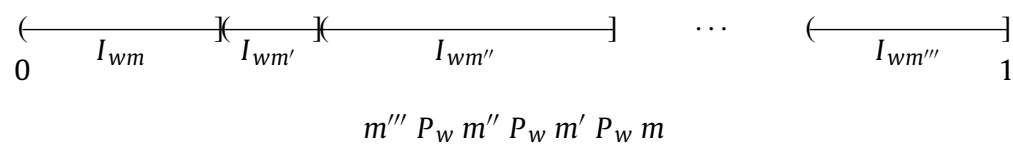

Due to Lemma 2 Part 2, for each pair $(m, w)$, the intervals $I_{m w}$ and $I_{w m}$ coincide. Thus, the following procedure gives a well-defined matching. Pick $x \in(0,1]$. For each pair $(m, w)$, match $m$ with $w$ if and only if $\pi_{m w}>0$ and $x \in I_{m w}$ [or equivalently $x \in I_{w m}$ ]. This matching is stable: for each $(m, w)$ such that $m$ prefers $w$ to his match, either $\pi_{m w}>0$ and $I_{m w}$ is on the left of $x$ and therefore $I_{w m}$ is on the left of $x$ and $w$ does not prefer $m$ to her match, or $\pi_{m w}=0$ and due to Lemma 2 Part $1, w$ does not prefer $m$ to her match. Now, if the real number $x$ is picked according to the uniform distribution on $(0,1]$, it is easy to see that we have a well-ordered decomposition of $\pi$.

Theorem 2. If an assignment is ex-post stable at a strict preference profile, then there is a utility profile $u$ consistent with the preference profile such that $\pi \in C\left(V^{u}\right)$.

Proof. Follows from Propositions 2 and 3.

The following example shows that the converse of Theorem 2 is not true. 
Example 2. Let $M=\{1,2,3\}$ and $W=\{a, b, c\}$. Let the preference profile $R$ and the utility profile $u$ that is consistent with $R$ be as follows.

\begin{tabular}{llllll}
\hline$R_{1}$ & $u_{1}$ & $R_{2}$ & $u_{2}$ & $R_{3}$ & $u_{3}$ \\
\hline$c$ & $\frac{2}{3}$ & $a$ & $\frac{2}{3}$ & $a$ & $\frac{1}{2}+\epsilon$ \\
$a$ & $\frac{1}{3}$ & $c$ & $\frac{1}{3}$ & $b$ & $\frac{1}{2}$ \\
$b$ & 0 & $b$ & 0 & $c$ & 0 \\
\hline
\end{tabular}

\begin{tabular}{llllll}
\hline$R_{a}$ & $u_{a}$ & $R_{b}$ & $u_{b}$ & $R_{c}$ & $u_{c}$ \\
\hline 1 & $\frac{2}{3}$ & 3 & $\frac{1}{2}$ & 2 & $\frac{2}{3}$ \\
3 & $\frac{1}{3}+\epsilon$ & 1 & $\frac{1}{3}+\epsilon$ & 3 & $\frac{1}{3}+\epsilon$ \\
2 & $\frac{1}{3}$ & 2 & $\frac{1}{3}$ & 1 & $\frac{1}{3}$ \\
\hline
\end{tabular}

Let $\mu$ be the assignment where 1 is matched with $a, 2$ with $c$, and 3 with $b$. Let $\mu^{\prime}$ be the assignment where 1 is matched with $c, 2$ with $a$, and 3 with $b$. Note that $(3, a)$ blocks $\mu^{\prime}$. Let $\pi$ be the assignment that assigns 0.5 probability to each of $\mu$ and $\mu^{\prime}$. Since this is the only possible decomposition of $\pi$, it is not ex-post stable.

Next, let $u$ be as described with the numerical values in the profile. Note that each man or woman receives the same expected utility $1 / 2$ from $\pi$ at $u$. Moreover, for each pair $(m, w) \in M \times W$, if $\pi_{m w}>0$ then $u_{m}(w)+u_{w}(m)=1$ and for a small enough choice of $\epsilon$, if $\pi_{m w}=0$ then $u_{m}(w)+u_{w}(m)<1$. It follows that $\pi \in C\left(V^{u}\right)$.

For deterministic assignments, if we restrict ourselves to strict preferences (at which no agent is indifferent between any two different agents), stability implies efficiency. To see this, suppose that a deterministic assignment is not efficient. Then there is another assignment at which an agent, say agent $i$, is better off, which means that $i$ is matched to agent $j$ whom he prefers to his/her current mate. Since $j$ cannot be worse off in the new assignment, $j$ prefers $i$ to his/her current mate. Thus, the initial assignment is not stable. However, once indifferences are allowed, there is no implication relation between efficiency and stability. ${ }^{15}$

As opposed to the deterministic case, in the probabilistic case, the relation between sd-efficiency and ex-post stability is not evident. If we allow for indifferences, since efficiency is not related to stability in the deterministic case, ex-post stability and sd-efficiency are not related either. However, if we restrict ourselves to strict preferences, the relation between ex-post stability and sd-efficiency is not so clear. Yet, it follows from Theorem 2 that ex-post stability implies sd-efficiency when preferences are strict. ${ }^{16}$

Corollary 2. If an assignment is ex-post stable at a strict preference profile, then it is sd-efficient at that preference profile.

Echenique and Galichon (2014) ask if there is a utility profile consistent with ordinal preferences such that each stable deterministic assignment is in the core of the associated TU-game. They provide the following partial answer. Define an isolated stable assignment as a stable deterministic assignment such that for each pair of matched agents, there is no other stable assignment at which they are matched to each other; then, there is a utility profile consistent with the ordinal preferences such that each isolated stable assignment is in the core. To see that this result also follows from our results, consider the ex-post stable assignment that is a uniform lottery over these isolated stable deterministic assignments. It is easy to see that this lottery is the unique decomposition of the ex-post stable assignment into stable deterministic assignments and therefore, the isolated stable deterministic assignments are well-ordered. Now, one can show that the utility profile that follows from our construction is such that not only the ex-post stable probabilistic assignment, but also each of these deterministic isolated stable assignments belongs to the core. It is simply due to the fact that for each isolated assignment, for each unmatched man-woman pair, the sum of the utility the man gets from his mate and the utility the woman gets from her mate is equal to 1. As Echenique and Galichon (2014) point out, it is not clear if this result can be generalized to the entire set of stable assignments. However, it is rather clear that one can construct a utility profile such that each stable assignment is utilitarian welfare-maximizing.

\section{Extensions of the model}

We present extensions of our model and discuss whether our results still follow for these extensions or not.

Allowing for remaining single: Suppose that each man or woman is allowed to remain single, and the preference relation of each $m \in M$ is a strict preference relation over $W \cup M$, where being assigned to $m$ represents remaining single, and for each $m^{\prime} \in M \backslash\{m\}$, we have for each $w \in W, w P_{m} m^{\prime}$ and $m P_{m} m^{\prime}$. We extend the preference relation of each $w \in W$ to $M \cup W$ similarly.

\footnotetext{
$\overline{15}$ Consider a serial dictatorship, which, according to a predetermined order, assigns each man to one of his most preferred women from among the remaining ones. One can easily specify preferences so that this produces an efficient but unstable assignment. To see that there is a stable but inefficient assignment, consider two men $\left\{m_{1}, m_{2}\right\}$ and two women $\left\{w_{1}, w_{2}\right\}$. Let preferences be such that each woman is indifferent between the two men, $m_{1}$ prefers $w_{1}$ to $w_{2}$, and $m_{2}$ prefers $w_{2}$ to $w_{1}$. Note that assigning $m_{1}$ to $w_{2}$, and $m_{2}$ to $w_{1}$ is stable but inefficient.

16 To see this note that for a probabilistic assignment $\pi$, if there is a utility profile $u$ such that $\pi \in C\left(V^{u}\right)$, then $\pi$ is utilitarian efficient at $u$. This result is independently reported also by Manjunath (2011) (Proposition 2), the proof of which relies on Lemma 2 (Roth et al., 1993) that we report in Section 3.3.
} 
Note that, the binary relations that we defined to characterize sd-efficiency are defined over $M \times W$. To extend our results related to sd-efficiency to the setup where agents can remain single, for each $m \in M$ and $w \in W$, we add the pairs $(m, m)$ and $(w, w)$ to $M \times W$ and define the relations over this extended set in the same way.

One can easily see that in this extended setting, the sd-efficiency of a probabilistic assignment is equivalent to the weak acyclicity of $\succeq_{(\pi, R)}$ defined over the extended set. Since for Theorem 1, our construction of the utility profile is based on this binary relation, the construction of the utility profile for this extended case follows similarly in which for each agent we also obtain a utility index for remaining single.

As for the extension of Theorem 2, the key is to note that for a given preference profile, by the Rural Hospital Theorem (Roth, 1986), at each stable matching, the same men and women are single. It follows that each ex-post stable probabilistic assignment induces a sub-matrix that is doubly-stochastic, and the same men and women that are single. Now, for the matched men and women, consider the utility profile obtained by our Theorem 2 . The only question is how to incorporate the utility of remaining single to this profile. Since the given assignment is ex-post stable, each agent prefers each other agent that he or she is matched with positive probability to being single. So, for each agent, let the utility he/she gets from remaining single be -1 . One can construct the rest of the utility profile in such a way that for each unmatched man-woman pair, the utilities they get from each other are less than 0. Hence, remaining single or the possibility of getting matched with agents who are single is not a threat for the given ex-post assignment to be in the core.

Many-to-one setting: Suppose that there is a set of firms, denoted by $F$, and a set of workers, denoted by $W$. Each firm $f \in F$ has a capacity $c_{f}$ such that $\sum_{f \in F} c_{f}=|W|$. Each worker has a strict preference relation over firms. We assume that each firm $f$ has responsive strict preferences over sets of workers, that is, for each $S \subset W$ and each pair of workers $w, w^{\prime} \in W \backslash S$, we have $S \cup\{w\} P_{f} S \cup\left\{w^{\prime}\right\}$ if and only if $w P_{f} w^{\prime}$.

In a deterministic assignment, each worker is assigned to a firm such that each firm is assigned its capacity number of workers. A probabilistic assignment is an $|W| \times|F|$ stochastic matrix where each row corresponds to a worker, each column corresponds to a firm, each row sum is equal to 1 , and each column sum is equal to the capacity of the corresponding firm (Birkhoff, 1946 and Von Neumann, 1953 extends to this setting). A deterministic assignment is ex-post stable if there is no worker-firm pair such that the worker prefers the firm to his assigned firm, and the firm prefers the worker to one of his assigned workers. A probabilistic assignment is ex-post stable, if it can be decomposed into stable deterministic assignments. As before, let $\Pi$ denote the set of all probabilistic assignments and let $\mathcal{P}$ denote the set of strict preference profiles.

We extend the notion of well-ordered ex-post stability to this setting in the following way. An assignment $\pi \in \Pi$ is many-to-one well-ordered ex-post stable at $\boldsymbol{P} \in \mathcal{P}$ if it has a decomposition into stable deterministic (many-to-one) assignments $\mu_{1}, \ldots, \mu_{T}$ such that for each $t, t^{\prime} \in\{1, \ldots, T\}$ with $t<t^{\prime}$, each worker either strictly prefers his assignment at $\mu_{t^{\prime}}$ to his assignment at $\mu_{t}$ or he is assigned to the same firm in both assignments, and each firm strictly prefers each worker that it receives at $\mu_{t}$ to each worker that it receives in $\mu_{t^{\prime}}$ but not in $\mu_{t}$, that is,

i. for each $w \in W, \mu_{t^{\prime}}(w) P_{w} \mu_{t}(w)$, and

ii. for each $f \in F$, for each $w \in \mu_{t}(f)$, and for each $w^{\prime} \in \mu_{t^{\prime}}(f) \backslash \mu_{t}(f)$, we have $w P_{f} w^{\prime}$.

Note that, given responsive preferences, each firm weakly prefers its set of workers at $\mu_{t}$ to its set of workers at $\mu_{t^{\prime}}$. We show that each many-to-one ex-post stable assignment has a many-to-one well-ordered stable decomposition, that is, Proposition 3 extends to this setting.

Proposition 4. If a many-to-one assignment is ex-post stable at a strict preference profile, then it has a many-to-one well-ordered stable decomposition.

Proof. Consider an ex-post stable assignment $\pi$ with a stable decomposition $\pi=\lambda_{1} \mu_{1}+\lambda_{2} \mu_{2}+\cdots+\lambda_{T} \mu_{T}$. Consider the following auxiliary one-to-one problem and the auxiliary assignment $\pi^{\prime}$ : for each firm $f$, there are $\left|c_{f}\right|$ copies, indexed from 1 to $\left|c_{f}\right|$, such that each copy has the same preference relation as the firm's original preference relation; each worker's preference relation is such that given two copies of firms, if the copies belong to different firms, then the worker's preference relation agrees with his original preference relation, and if the two copies belong to the same firm, then the worker prefers the copy with the lower index to the other copy. Note that the auxiliary problem belongs to our one-to-one setting. For each $\mu_{t}$ where $t \in\{1, \ldots, T\}$, let $\mu_{t}^{\prime}$ be the deterministic assignment in the one-to-one setting with copies such that each worker is assigned to a copy of the firm that he is assigned to at $\mu_{t}$, and for each pair of copies of the same firm, if the copy with a higher index is assigned a worker, then the copy with a lower index is assigned a more preferable worker.

Let $\pi^{\prime}=\lambda_{1} \mu_{1}^{\prime}+\lambda_{2} \mu_{2}^{\prime}+\cdots+\lambda_{T} \mu_{T}^{\prime}$. It is easy to see that $\pi^{\prime}$ is ex-post stable at the auxiliary one-to-one problem. Then, by Proposition 3, it has a well-ordered stable decomposition $\pi^{\prime}=\lambda_{1}^{\prime} \mu_{1}^{\prime \prime}+\lambda_{2}^{\prime} \mu_{2}^{\prime \prime}+\cdots+\lambda_{K}^{\prime} \mu_{K}^{\prime \prime}$ such that for each $t$, $t^{\prime}$ with $1 \leq t<t^{\prime} \leq K$, each copy of any firm weakly prefers $\mu_{t}$ to $\mu_{t^{\prime}}$ and each worker weakly prefers $\mu_{t^{\prime}}$ to $\mu_{t}$. Now, for each $t \in\{1, \ldots, K\}$, let $\bar{\mu}_{t}$ be the many-to-one deterministic assignment where a worker $w$ is assigned to a firm $f$ if and only if $w$ is assigned to a copy of $f$ at $\mu_{t}^{\prime \prime}$. Clearly, for each $t \in\{1, \ldots, K\}, \bar{\mu}_{t}$ is stable and $\pi=\lambda_{1}^{\prime} \bar{\mu}_{1}+\lambda_{2}^{\prime} \bar{\mu}_{2}+\cdots+\lambda_{K}^{\prime} \bar{\mu}_{K}$.

We show that $\pi=\lambda_{1}^{\prime} \bar{\mu}_{1}+\lambda_{2}^{\prime} \bar{\mu}_{2}+\cdots+\lambda_{K}^{\prime} \bar{\mu}_{K}$ is a many-to-one well-ordered stable decomposition of $\pi$. Let $t, t^{\prime} \in$ $\{1, \ldots, K\}$ such that $t<t^{\prime}$. By construction, for each $w \in W, \mu_{t^{\prime}}(w) P_{w} \mu_{t}(w)$. Let $f \in F, w \in \mu_{t}(f)$, and $w^{\prime} \in \mu_{t^{\prime}}(f) \backslash$ $\mu_{t}(f)$. Suppose that $w^{\prime} P_{f} w$. Since $f=\mu_{t^{\prime}}\left(w^{\prime}\right) P_{w^{\prime}} \mu_{t}\left(w^{\prime}\right)$, we have a contradiction to $\bar{\mu}_{t}$ being stable. Thus $w P_{f} w^{\prime}$, which completes the proof. 
Although the well-ordered stable decomposition result (Proposition 3) extends to the many-to-one setting, whether Proposition 2 extends is not clear. To see why a construction similar to the one in the proof of Proposition 2 does not work, consider a firm $f$ that is matched with workers $w$ and $w^{\prime}$ with positive probabilities. Suppose that $f$ prefers $w$ to $w^{\prime}$. To sustain the core property, we should keep each admissible coalition's sum of expected utilities from the given assignment above the value of that coalition. To do so, similar to our earlier construction, we can define the utility that $f$ gets from $w$ as a convex combination of the utility indexes associated with the assignments that $f$ is assigned to $w$ in the well-ordered decomposition. Now, although the first stable assignment in the decomposition that $f$ is assigned to $w$ precedes (or is the same as) that of $w^{\prime}$, this may not be true for the last stable assignment that $f$ is assigned to $w$. Therefore the utility that $f$ gets from $w$ can be less than the utility that $f$ gets from $w^{\prime}$, whence the utility profile that we construct may not be consistent with the given preference profile. ${ }^{17}$ Such a problem never arises in the one-to-one setting since a man cannot be matched with two women in the same assignment. To sum up, it is not clear to us whether Proposition 2 and Theorem 2 extend to the many-to-one setting or not, and we leave this problem for future research.

\section{Conclusion}

Marriage problems constitute a basis for many real-life economic problems. Here, we considered the efficiency and stability of probabilistic assignments in marriage problems when only ordinal preference information is available. When we only have ordinal preference information, two common ways to evaluate probabilistic assignments in terms of efficiency and stability are sd-efficiency and ex-post stability, respectively. We asked whether probabilistic assignments that are sdefficient or ex-post stable for the ordinal preferences are possibly efficient or stable for the cardinal preferences. Our answer was positive. Of course, this positive answer does not provide a strong justification for the efficiency and stability notions mentioned above, as there may be several utility profiles consistent with the ordinal preferences for which the sd-efficient or ex-post stable assignments are not desirable. However, our results show that there is at least one utility profile for which these assignments continue to be desirable, which provides a further understanding of the structure of such assignments and the connection between efficiency and stability notions in the ordinal and cardinal environments.

\section{References}

Athanassoglou, S., 2011. Efficiency under a combination of ordinal and cardinal information on preferences. J. Math. Econ. 47, 180-185. Aziz, H., Brandl, F., Brandt, F., 2015. Universal Pareto dominance and welfare for plausible utility functions. J. Math. Econ. 60, 123-133. Becker, G.S., 1973. A theory of marriage: part I. J. Polit. Economy 81 (4), 813-846.

Birkhoff, G., 1946. Three observations on linear algebra. Rev. Univ. Nac. Tucuman, Ser. A 5, 147-151.

Bogomolnaia, A., Moulin, H., 2001. A new solution to the random assignment problem. J. Econ. Theory 100, 295-328.

Bogomolnaia, A., Moulin, H., 2004. Random matching under dichotomous preferences. Econometrica 72, 257-279.

Carroll, G., 2011. An efficiency theorem for incompletely known preferences. J. Econ. Theory 145, 2463-2470.

Echenique, F., 2008. What matchings can be stable? The testable implications of matching theory. Math. Oper. Res. 33 (3), 757-768.

Echenique, F., Galichon, A., 2014. Ordinal and cardinal solution concepts for two-sided matching. Available at SSRN 2246031.

Echenique, F., Lee, S., Shum, M., Yenmez, M.B., 2013. The revealed preference theory of stable and extremal stable matchings. Econometrica 81 (1), $153-171$. Gale, D., Shapley, L.S., 1962. College admissions and the stability of marriage. Amer. Math. Mon. 69 (1), 9-15.

Katta, A.K., Sethuraman, J., 2006. A solution to the random assignment problem on the full preference domain. J. Econ. Theory 131, 231-250.

Kesten, O., Ünver, U., 2015. A theory of school choice lotteries. Theoretical Econ. 10, 543-595.

Knuth, D., 1976. Marriages Stables. Montreal University Press.

Manea, M., 2008. A constructive proof of the ordinal efficiency welfare theorem. J. Econ. Theory 141, 276-281.

Manjunath, V., 2011. Stability and the core of probabilistic marriage problems. Available at SSRN 1809941.

Manjunath, V., 2014. Markets for fractional partnerships. Available at SSRN 1809968.

McLennan, A., 2002. Ordinal efficiency and the polyhedral separating hyperplane theorem. J. Econ. Theory 105, 435-449.

Roth, A.E., 1986. On the allocation of residents to rural hospitals: a general property of two-sided matching markets. Econometrica 54, 425-427.

Roth, A., Sotomayor, M., 1990. Two-Sided Matching: A Study in Game-Theoretic Modeling and Analysis. Econometric Society Monographs. Cambridge University Press, Cambridge, UK.

Roth, A.E., Uriel, G.R., Vande Vate, J.H., 1993. Stable matchings, optimal assignments, and linear programming. Math. Oper. Res. 18 (4), $803-828$.

Rothblum, U., 1992. Characterization of stable matchings as extreme points of a polytope. Math. Program. 54, 57-67.

Shapley, L., Shubik, M., 1971. The assignment game I: the core. Int. J. Game Theory 1 (1), 111-130.

Teo, C.P., Sethuraman, J., 1998. The geometry of fractional stable matchings and its applications. Math. Oper. Res. 23 (4), 874-891.

Thomson, W., 2010. Strategy-proof allocation rules. University of Rochester. Mimeo.

Vande Vate, J.H., 1989. Linear programming brings marital bliss. Oper. Res. Lett. 8, 147-153.

Von Neumann, J., 1953. A certain zero-sum two-person game equivalent to the optimal assignment problem. In: Kuhn, H.W., Tucker, A.W. (Eds.), Contributions to the Theory of Games. Princeton University Press, Princeton, NJ.

\footnotetext{
17 We encounter a similar problem when we attempt to construct a utility profile for a firm $f$ by aggregating the utility functions of the copies of $f$ constructed in the proof of Proposition 4.
} 